\title{
Ultrasound 2020 - Diagnostics \& Therapy: On the Way to Multimodal Ultrasound: Contrast-Enhanced Ultrasound (CEUS), Microvascular Doppler Techniques, Fusion Imaging, Sonoelastography, Interventional Sonography
}

\author{
Ultraschall 2020 - Diagnostik \& Therapie: Auf dem Weg zur \\ multimodalen Sonografie: kontrastverstärkter Ultraschall (CEUS), \\ mikrovaskuläre Dopplerverfahren, Fusionsbildgebung, \\ Sono-Elastografie, interventionelle Sonografie
}

Authors

Christopher Kloth ${ }^{1}$, Wolfgang Kratzer ${ }^{2}$, Julian Schmidberger ${ }^{2}$, Meinrad Beer ${ }^{1}$, Dirk Andre Clevert ${ }^{3}$, Tilmann Graeter ${ }^{1}$

Affiliations

1 Department of Interventional and Diagnostic Radiology, University Hospital Ulm, Germany

2 Department of Internal Medicine I, University Hospital Ulm, Germany

3 Department of Clinical Radiology, University Hospital Munich Campus Großhadern, München, Germany

Key words

B-scan, contrast-enhanced ultrasound, elastography, interventional, doppler ultrasonography

received 10.02.2020

accepted 21.06.2020

published online 30.07 .2020

Bibliography

Fortschr Röntgenstr 2021; 193: 23-32

DOI 10.1055/a-1217-7400

ISSN 1438-9029

(c) 2020. Thieme. All rights reserved.

Georg Thieme Verlag KG, Rüdigerstraße 14,

70469 Stuttgart, Germany

Correspondence

Dr. Tilmann Graeter

Department of Diagnostic and Interventional Radiology, University Hospital Ulm, Albert-Einstein-Allee 23, 89081 Ulm, Germany

Tel.: ++49/7 31/50061175

tilmann.graeter@uniklinik-ulm.de

\section{ABSTRACT}

Background Ultrasound as a non-ionizing imaging procedure is one of the most important diagnostic procedures in everyday clinical practice. The technology is widely used. Due to constant technical innovations, sonographic procedures, such as contrast-enhanced ultrasound (CEUS), sonoelastogra- phy, new microvascular Doppler modalities and, as an example of interventional procedures, sonographically controlled microwave ablation (MWA), are becoming increasingly important in diagnostic imaging and interventional medicine alongside CT and MRI. However, this also requires greater expertise, specialization and qualification on the part of users.

Method This review article provides information about the range of technical innovations in ultrasound in recent years and describes the underlying technology, the clinical applications, and their diagnostic value. These are presented in the context of the current literature, explaining their advantages and disadvantages and their clinical value.

Results and Conclusion Contrast-enhanced ultrasound (CEUS), microvascular Doppler modalities, fusion imaging and elastography complement B-scan ultrasound and conventional Doppler procedures for various problems. Microwave ablation (MWA) has a firm place as an ablative procedure for local tumor therapy in different organ systems and can be performed under ultrasound guidance. Thanks to new developments, the possibilities of ultrasound are now greater than ever. Knowledge of the technology, indications, and possible applications of newer procedures is essential for adequate patient care.

Key Points:

- Contrast-enhanced ultrasound (CEUS) allows an increase in sensitivity and specificity in the assessment of parenchymal lesions.

- CEUS allows the microperfusion to be visualized and quantified. For larger vessels, CEUS is an important instrument in diagnosing endoleak after stenting.

- Microvascular Doppler techniques with clutter suppression algorithms allow a more accurate representation of the smallest vessels than regular color or power Doppler.

- Elastography of the liver in diffuse hepatopathies is a noninvasive diagnostic tool to exclude higher grade fibrosis/ cirrhosis. 
- Microwave ablation (MWA) also offers sonographically controlled ablation of tumors.

\section{Citation Format}

- Kloth C, Kratzer W, Schmidberger J et al. Ultrasound 2020 - Diagnostics \& Therapy: On the Way to Multimodal Ultrasound: Contrast-Enhanced Ultrasound (CEUS), Microvascular Doppler Techniques, Fusion Imaging, Sonoelastography, Interventional Sonography . Fortschr Röntgenstr 2021; 193: 23-32

\section{ZUSAMMENFASSUNG}

Hintergrund Ultraschall als nichtionisierendes bildgebendes Verfahren stellt eines der wichtigsten diagnostischen Verfahren im Klinikalltag dar. Bei weiter Verbreitung findet es flächendeckenden Einsatz. Durch stetige technische Innovationen gewinnen sonografische Verfahren wie kontrastverstärkter Ultraschall (CEUS), Sono-Elastografie, neue mikrovaskuläre Dopplermodalitäten und als Beispiel für interventionelle Verfahren die sonografisch gesteuerte Mikrowellenablation (MWA) zunehmend an Bedeutung innerhalb der bildgebenden Diagnostik und Intervention neben der CT- und MRT-Schnitt- bildtechnik. Dies erfordert jedoch auch eine höhere Expertise, Spezialisierung und Qualifikation bei den Anwendern.

Methode Dieser Übersichtsartikel informiert über das Spektrum technischer Neuerungen im Ultraschall der letzten Jahre und beschreibt die zugrunde liegende Technik, die klinischen Anwendungsmöglichkeiten und deren diagnostischen Stellenwert. Diese werden unter Darlegung von Vor- und Nachteilen sowie ihrer klinischen Wertigkeit im Kontext der aktuellen Literatur vorgestellt.

Ergebnisse und Schlussfolgerung Die Verfahren des kontrastverstärkten Ultraschalls (CEUS), mikrovaskulärer Dopplermodalitäten, der Fusionsbildgebung und der Elastografie ergänzen den B-Bild-Ultraschall und konventionelle DopplerVerfahren bei unterschiedlichen Fragestellungen. Die Mikrowellenablation (MWA) hat einen festen Stellenwert als ablatives Verfahren zur lokalen Tumortherapie an verschiedenen Organsystemen und kann ultraschallgesteuert erfolgen. Die Möglichkeiten des Ultraschalls sind dank neuer Entwicklungen heutzutage größer denn je. Die Kenntnis über Technik, Indikationen und Anwendungsmöglichkeiten neuerer Verfahren sind für eine adäquate Patientenversorgung obligat.

\section{Introduction}

One reason for the growing importance of ultrasound and the increase in possible applications is the establishment of new modalities that have shifted in recent years from use purely for research to use in the clinical routine. In particular, sonoelastography and contrast-enhanced ultrasound (CEUS) have a number of well-established diagnostic applications. The development of new ultrasound methods with increasing technological diversity presents new challenges for radiologists. Diagnostic ultrasound imaging is an integral part of radiology training. However, it is currently established in only $78.5 \%$ of radiology departments as a routine diagnostic imaging method, while contrast-enhanced ultrasound is established in only $26 \%$. A possible solution here could be interdisciplinary ultrasound centers. In particular, training and continuing education can be better organized in combination and the use of high-end devices can be additionally optimized [2].

Knowledge of the technical principles of new ultrasound modalities, their possible applications in various organ systems, and their limitations is necessary to ensure optimal use of diagnostic ultrasound imaging. In addition to purely diagnostic modalities, local ablative procedures using ultrasound as part of tumor therapy have also become more diverse in recent years and have been included in guidelines. It is essential to know the strengths and weaknesses of each local ablative method in order to be able to select the best method for a particular case. An example of this is the use of ultrasound-guided microwave ablation (MWA) in large tumor lesions that may not be able to be sufficiently covered by radiofrequency ablation.

Fusion imaging makes it possible to combine real-time ultrasound examination with previously acquired CT or MRI datasets.
As a result, lesions that are difficult to access or are very small can be biopsied better under ultrasound guidance or a more precise differential diagnosis of lesions can be achieved using a multimodal approach.

The various new ultrasound modalities are presented in the following with respect to their current clinical significance in the individual areas of application.

\section{Contrast-enhanced ultrasound (CEUS)}

\section{Technique}

Microbubbles are administered intravenously in contrast-enhanced ultrasound (CEUS). The microbubbles are a few $\mu \mathrm{m}$ in size and oscillate after longitudinal ultrasound wave excitation in the examination field at a low mechanical index. The oscillating microbubbles generate nonlinear oscillations. The received contrast signal can be differentiated from the tissue signal by corresponding contrast agent software. Using real-time imaging software, organ vascularization can be visualized and also quantified. In contrast to contrast agents used in CT/MRI, the ultrasound contrast agent primarily used in Germany(SonoVue ${ }^{\circledR}$, Bracco, Milano, Italy) is a purely intravascular contrast agent. Thus, the ultrasound contrast agent primarily used in Germany is not absorbed by body cells [3]. Given the high percentage of gas, the ultrasound contrast agent is primarily eliminated via the lungs. The small amount of bubble shells is eliminated by the hepatobiliary system. Therefore, application in patients with limited renal function is possible. The risk of serious adverse events is very low at less than $0.008 \%$ [4]. 


\section{Clinical applications}

CEUS is most commonly used to examine the organs of the upper abdomen, mainly the liver and kidneys ( $\triangleright$ Fig. 1). While B-mode ultrasound in combination with color Doppler is often sufficient to characterize simple cysts and typical liver hemangiomas, CEUS can be used to characterize all other unclear focal and extensive changes in the liver with a sensitivity of over $90 \%$ [5]. Characterization is based on the contrast agent behavior in the arterial phase (up to approx. 25 seconds after injection), in the portal venous phase (starting approx. 25-45 seconds after injection) and in the late phase (starting 2 minutes after injection). The type of wash-in and the degree of contrast enhancement in the arterial phase and the contrast behavior in the venous and the late phase provide important information regarding the benign/malignant status of a liver lesion and the liver tumor entity. For example, washout over the course of imaging is an important sign of malignancy. For example, particularly late washout with significant arterial enhancement and a chaotic vascular pattern is typical for hepatocellular carcinoma (HCC).

Cystic renal lesions are common incidental findings on $\mathrm{CT}$ and MRI but often cannot be sufficiently characterized, for example, by single-phase CT. An increase in size or protein-rich deposits are common even in simple cysts but can result in uncertainty when interpreting results. The first step to clarify here is B-mode ultrasound and color Doppler. Vessels should not be visible within a simple renal cyst on color-coded duplex ultrasound since the presence of vessels indicates solid tissue. CEUS allows more precise evaluation of possible vascularization and the vascularization pattern, e. g. in internal septa, in real time allowing better evaluation of the malignancy risk of cystic as well as solid renal lesions [6]. However, since CEUS is significantly more sensitive than CT with respect to weak blood flow, e. g. in septa or in the cystic wall, "upgrading" of the Bosniak classification can occur which then affects the specificity. The high sensitivity of CEUS with respect to weak blood flow can result in even minimally vascularized papillary renal carcinomas being better detected with this modality than with CT.

In addition to the organs of the upper abdomen, CEUS can also be used, for example, in the skeletal musculature to visualize and quantify microperfusion [7]. The studies by Fischer et al. and Doll et al. with a significantly better differentiation between septic and non-septic impaired fracture healing show an innovative application of CEUS. The sensitivity and specificity are $85.1 \%$ and $88.7 \%$, respectively $[8,9]$. As shown by Marcon et al., CEUS is also increasingly important for answering questions regarding the testicles in testicular trauma, suspicion of testicular torsion, scrotal infections, or testicular tumors [10]. For example, it allows significantly better differentiation between a simple testicular cyst and a cystic testicular tumor with partial perfusion. The results of multiple studies regarding contrast behavior in the differentiation of seminomas from Leydig cell tumors are not definitive even though fast wash in and washout must be assessed as a sign of malignancy. In the case of testicular laceration caused by trauma, CEUS can be used to differentiate areas with better perfusion from those with less perfusion, thereby contributing to testis-sparing surgery.

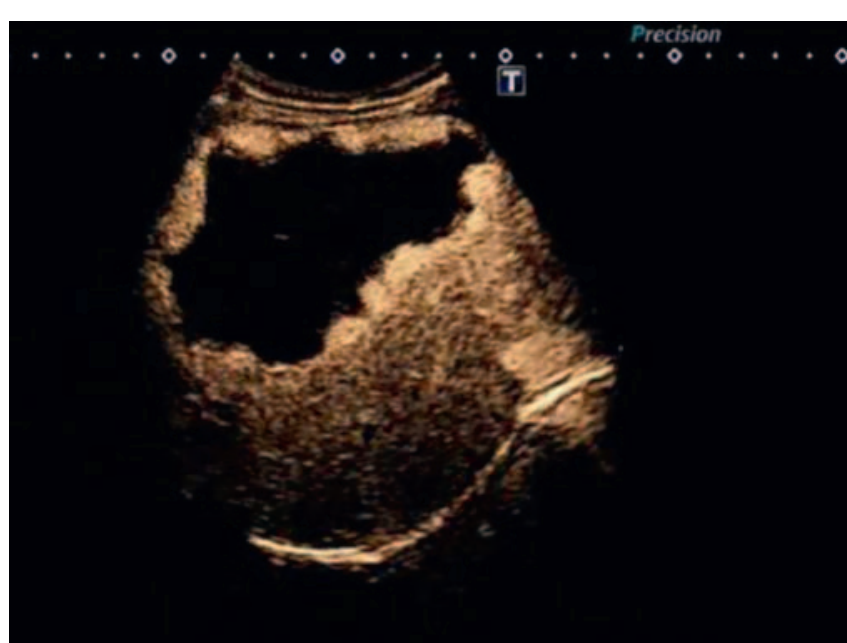

- Fig. 1 Giant hemangioma with central thrombosis with typical nodule-like rim enhancement in contrast-enhanced ultrasound (CEUS).

In addition, for years CEUS has been an important instrument in endoleak diagnosis after stent placement in large vessels. In recent years, CEUS has become established in the detection and characterization of endoleaks in the clinical routine and represents an important diagnostic method initially and during followup. In many cases, it can replace CT imaging which requires irradiation and nephrotoxic X-ray contrast media [11].

\section{Microvascular Doppler methods with interference suppression algorithm}

\section{Technique}

In contrast to regular color and power Doppler, the latest microvascular Doppler modalities can be used to visualize even small vessels with low flow rates. The technical approach is the expanded suppression of interference signals so that weak flow signals can also be detected and this information can be provided with a high image refresh rate [12]. Minimal flow can be shown in the form of a color overlay image or based on grayscales with subtraction of the underlying B-mode image.

\section{Clinical applications}

Microvascular Doppler methods with an interference suppression algorithm can be used, for example, to detect and evaluate the vascularization of parenchymatous lesions [13] ( Fig. 2). These methods are primarily used for oncological issues with parallels to CEUS application areas, e.g., for primary diagnosis or for follow-up during therapy. For example, Dubinsky et al. show that microvascular Doppler methods are superior to color/power Doppler for the evaluation of the vascularization of small HCC lesions of the liver $(<2 \mathrm{~cm})$ [14]. Compared to conventional Doppler modalities, microvascular Doppler methods are superior with respect to the evaluation of the benign/malignant status of cervical lymph nodes [15] as well as in the case of renal lesions like renal cell car- 


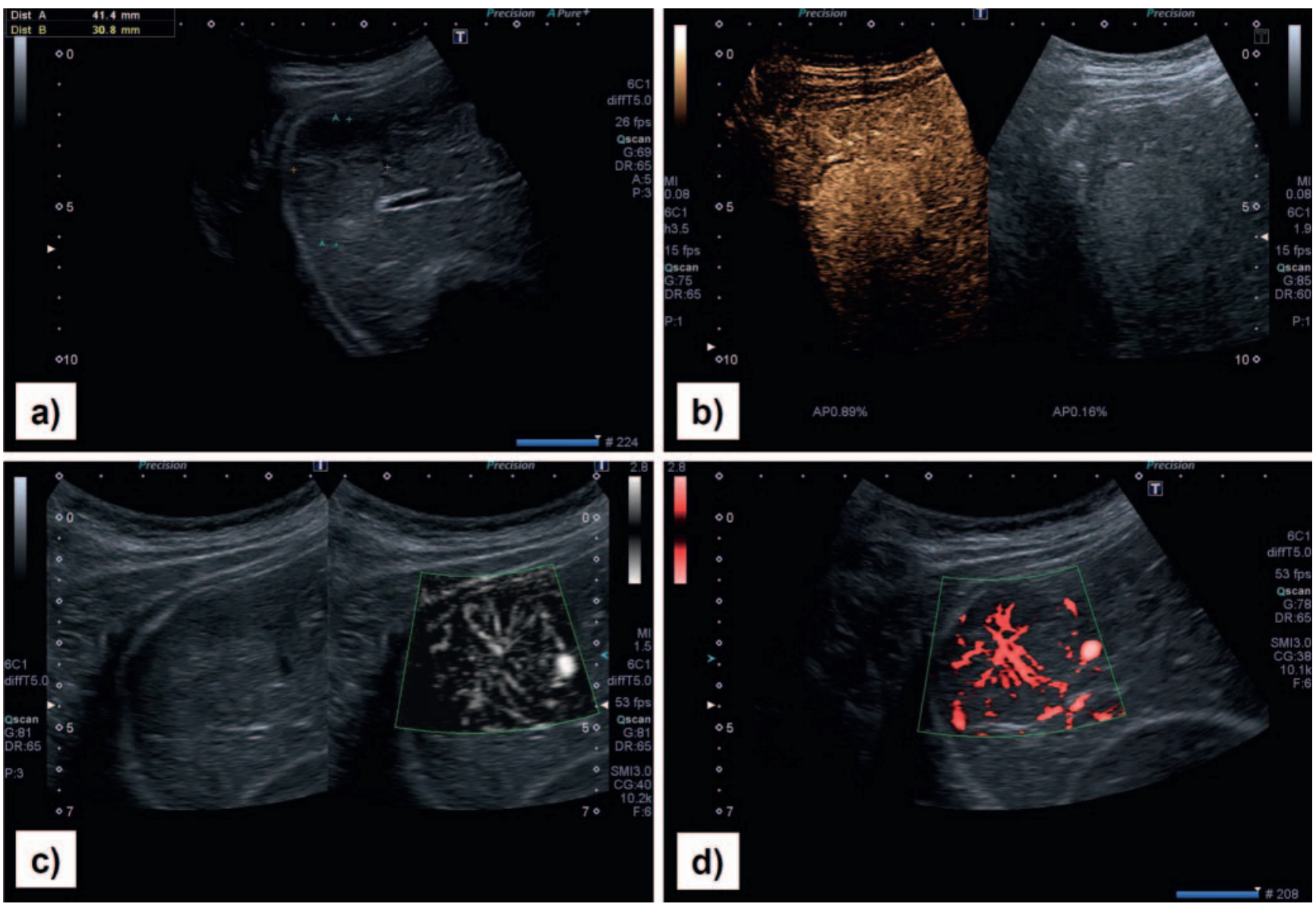

- Fig. 2 Illustration of a typical focal nodular hyperplasia (FNH) in a young female patient in the different modes. a B-scan display, b contrast-enhanced ultrasound arterial phase (CEUS), c microvascular Doppler technique monochrome), $\mathbf{d}$ microvascular Doppler technique color.

cinomas [16] or with respect to the vascularization of complex cysts [17].

There are a number of studies comparing microvascular Doppler methods to regular color/power Doppler or CEUS. Microvascular Doppler methods and CEUS have comparable sensitivity with respect to differentiating benign from malignant thyroid nodules. For microvascular Doppler methods, Gabriel et al. show comparable applicability, sensitivity, and reproducibility in the diagnosis of endoleaks after "endovascular aneurysm repair" (EVAR) compared to CEUS and CT angiography [19]. Microvascular Doppler methods performed sequentially in combination with regular Doppler methods has a diagnostic benefit also in acute diagnosis as in the diagnosis of acute cholecystitis [20]. However, numerous studies on microvascular Doppler methods also reference application areas outside of the abdomen: Park et al. showed the superiority of microvascular Doppler methods compared to conventional Doppler modalities regarding the detection of the vascularization of breast tumors [21] or in carpal tunnel syndrome [22]. The precise visualization of the smallest vessels by microvascular Doppler methods - particularly in monochrome mode - was confirmed also in the examination of the testicles to evaluate vascularization of the parenchyma [23].

There are numerous applications of microvascular Doppler methods in children and especially in newborns. As an example, microvascular Doppler methods can be used to evaluate the vascularization of the testicles or to evaluate the perfusion of the brain parenchyma [12].

A disadvantage of microvascular Doppler methods is that to date there is no quantification method and the degree of vascularization can only be provided in the form of semiquantitative scores [24].

\section{Fusion imaging}

\section{Technique}

For several years, it has been possible to fuse CT or MRI datasets with real-time ultrasound examinations. A CT or MRI examination stored in DICOM format is imported to an ultrasound device. After coupling of the datasets with the probe position in the region of corresponding organ structures on three spatial planes, both the ultrasound image in real time and the CT/MRI image can be viewed and evaluated in parallel ( $\downarrow$ Fig. 3 ). There are numerous possible applications, particularly in the characterization of parenchymal lesions as well as during interventions [25]. Ultrasound compensates for the limitations of computed tomography like 

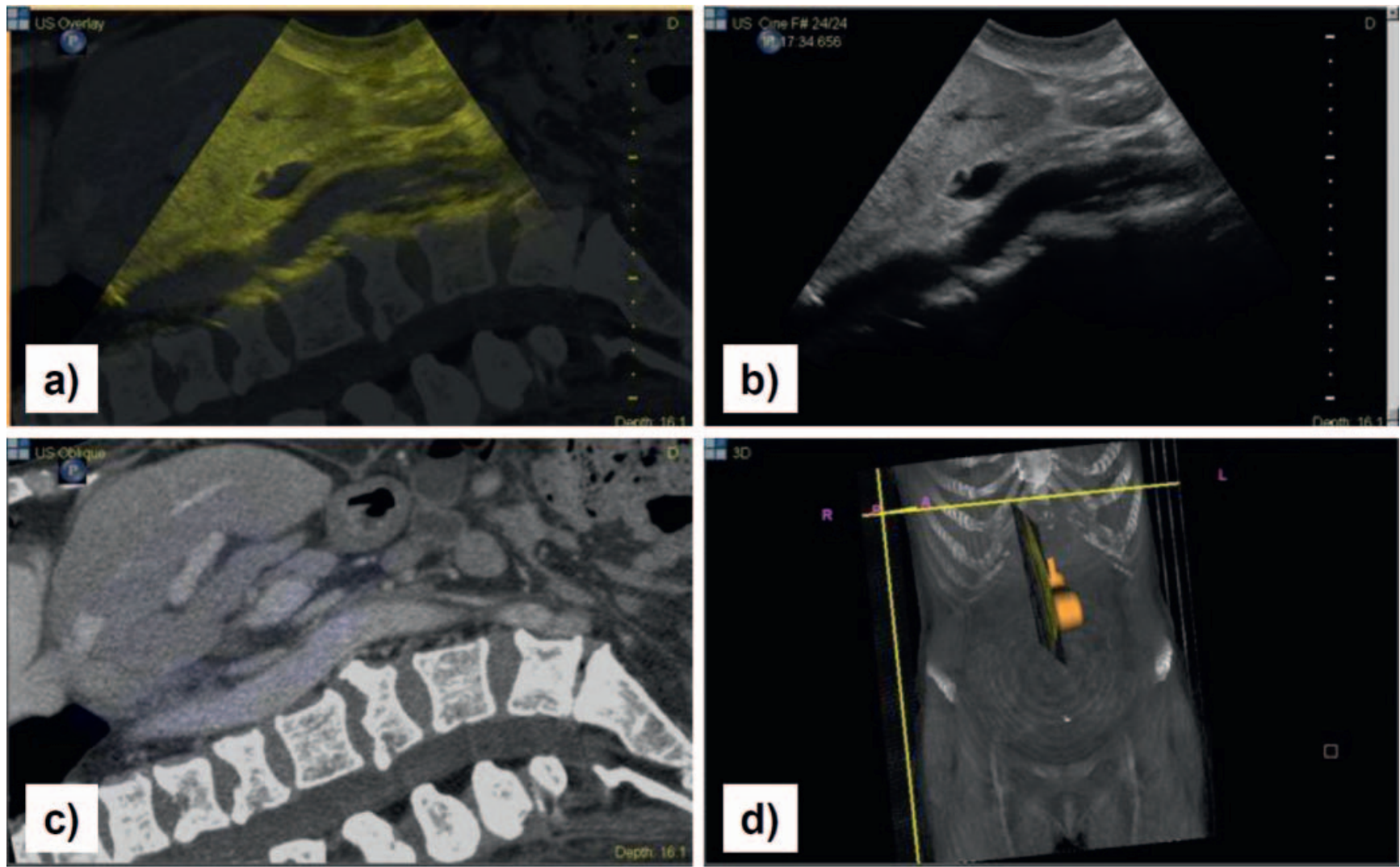

- Fig. 3 Representation of an upper abdominal situs in fusion mode, longitudinal section on the B-scan and in computed tomography (CT). a Representation of the superimposition of CT and sonography, $\mathbf{b}$ Representation on the B-image, $\mathbf{c}$ Representation on CT, $\mathbf{d}$ Position of the transducer in relation to the trunk.

partial volume effects, respiratory motion artifacts, and phasebased contrast enhancement.

\section{Clinical applications}

Successful image fusion has been discussed in detail in multiple studies: For example, Ewertsen et al. were able to show that unclear lesions were easier to characterize via image fusion between ultrasound and CT/MRI and that the benign/malignant status of a greater number of lesions can then be clarified. In addition to image fusion with CT and MRI datasets, PET-CT data can also be used [26]. Fusion imaging is primarily used for oncological applications like the detection and diagnosis of liver lesions in the clinical routine. Particularly in the case of a small lesion size of less than $10 \mathrm{~mm}$, the use of real time ultrasound-guided fusion imaging offers advantages with respect to detection on the B-mode image. Okamoto et al. were able to show in this context that smaller parenchymal lesions missed by regular B-mode ultrasound can be detected by fusion imaging [27]. An advantage of fusion imaging with respect to the detection rate of renal lesions was also able to be shown [28]. Interventional methods and biopsies represent a further field of application [29]. Fusion imaging expands the possibilities with respect to planning, implementation, and measurement of results. The most common application in this connection is puncture or ablation of lesions that are difficult to detect on ultrasound or are very small and suspicious for tumor [29-
31]. Tumor areas that are still vital after intervention, their precise anatomical surroundings, e. g. adjacent vessels, gas formation during treatment, or subsequent bleeding can be better detected via fusion imaging. In addition, limitations that can arise as a result of movement of the liver during respiration can be compensated at least partially by ultrasound image fusion [32]. A further application area of fusion imaging is the diagnosis of prostate cancer. As a result of the fusion of MRI and various ultrasound modalities, the accuracy of targeted transrectal prostate biopsies can be increased and an initial evaluation of the aggressiveness of prostate cancer can be performed [33].

While image fusion was associated with a significant time requirement in the early years, technical developments now allow quick application in the clinical routine. With the establishment of new cross-sectional imaging methods, e. g. PET-MRI, fusion imaging can be expected to become increasingly important in the future.

\section{Elastography}

\section{Technique}

In recent years, multiple ultrasound-based elastography methods for noninvasive determination of tissue elasticity have been developed. Their primary clinical application is the diagnosis and stag- 

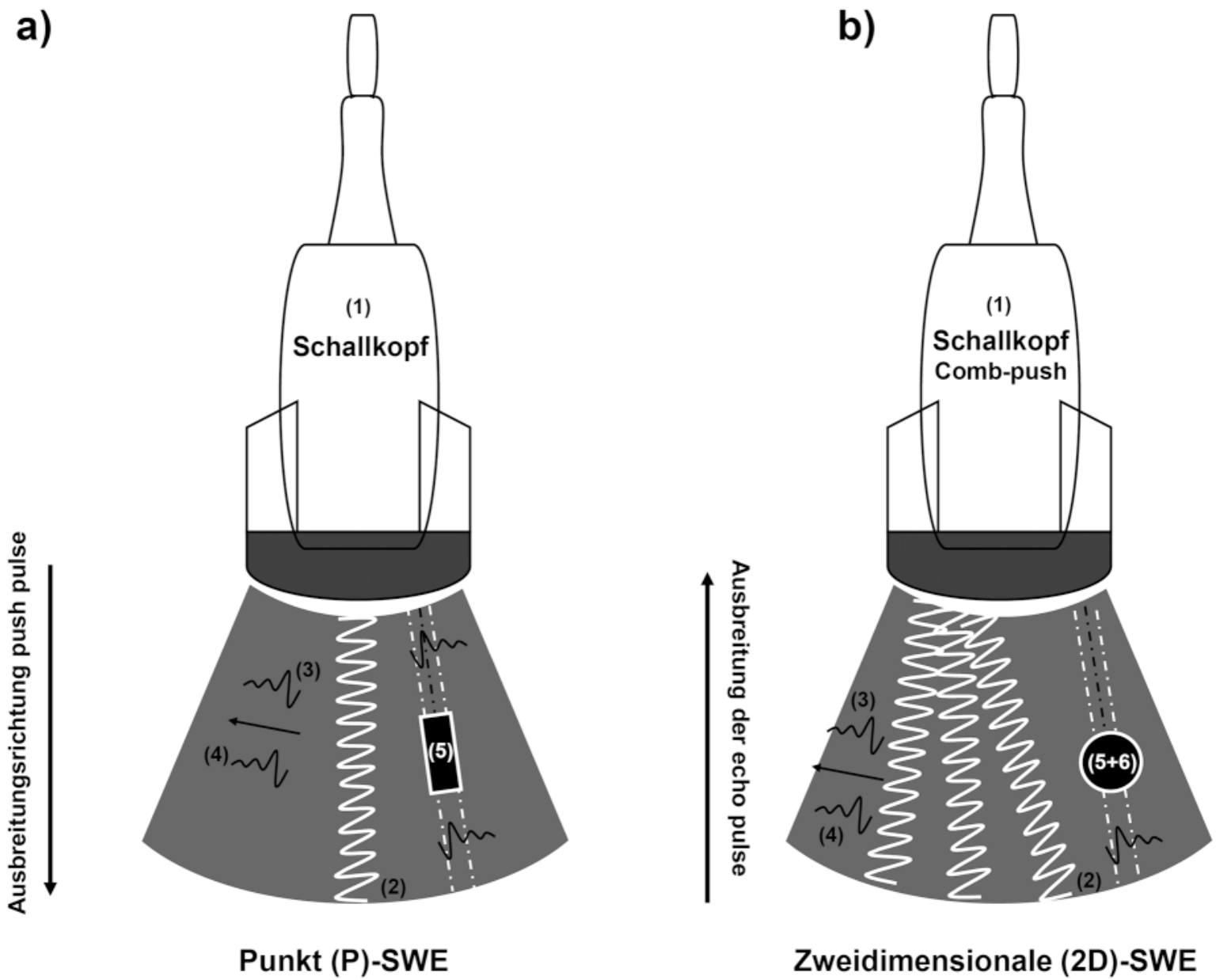

\section{Zweidimensionale (2D)-SWE}

- Fig. 4 Shear wave elastography (SWE). 1 Ultrasonic probe (transducer), 2 Push pulses (PI), 3 Shear waves (SW), 4 Horizontal propagation of sound waves, $\mathbf{5}$ Region of interest (ROI) in which ultrasonic waves measure the propagation speed of the sound waves, $\mathbf{6}$ Parallel transmitted image lines $(\mathrm{P})$.

ing of fibrotic liver diseases and they supplement and sometimes even replace the results of the current diagnostic gold standard, liver biopsy. The first method used in this area was transient elastography (TE). Although it has become established globally as a reliable non-imaging method for determining liver elasticity, it has various limitations including high dependence on the type of examination [34]. A new generation of elastography methods that do not depend on mechanical impulses for generating shear waves but rather use high-intensity ultrasound waves is collectively referred to as point shear wave elastography (pSWE). With similar or even superior diagnostic precision, the point shear wave methods are more practical than TE and also yield valid measurements in patients with a high body mass index (BMI) or ascites [35]. Moreover, as imaging methods they have the advantage that the examiner can use the anatomical B-mode scan to select a suitable location in the tissue for determining elasticity [36]. Some point shear wave methods can quantify tissue elasticity indirectly from the velocity of the induced shear waves. The point shear wave elastography method allows quantification of the shear wave velocity and is capable of providing a single value in $\mathrm{m} / \mathrm{s}$ or $\mathrm{kPa}$ in a field selected via B-mode imaging.

\section{Clinical applications}

Multiple studies have confirmed that the pSWE technique/virtual TouchTM tissue quantification (VTQ) is a valid staging method in liver fibrosis: A meta-analysis of eight studies with the AUROC (area under the receiver operating curve) yielded an accuracy of 0.87 for VTQ for significant liver fibrosis and 0.93 for cirrhosis, with liver biopsy being used as the reference standard [37]. 2D shear wave elastography (2D SWE) allows a very high frame rate in real-time images of shear wave propagation in a focused area. The result is a two-dimensional, color-coded elastogram in which a measurement field can be placed to detect quantitative data [36]. The most studied 2D-SWE method is the ultrasound shear imaging technique (SSI, Aixplorer ${ }^{\circledR}$ ) [38]. Other manufacturers have increasingly integrated elastography methods in their ultrasound devices. However, the data currently available regarding 

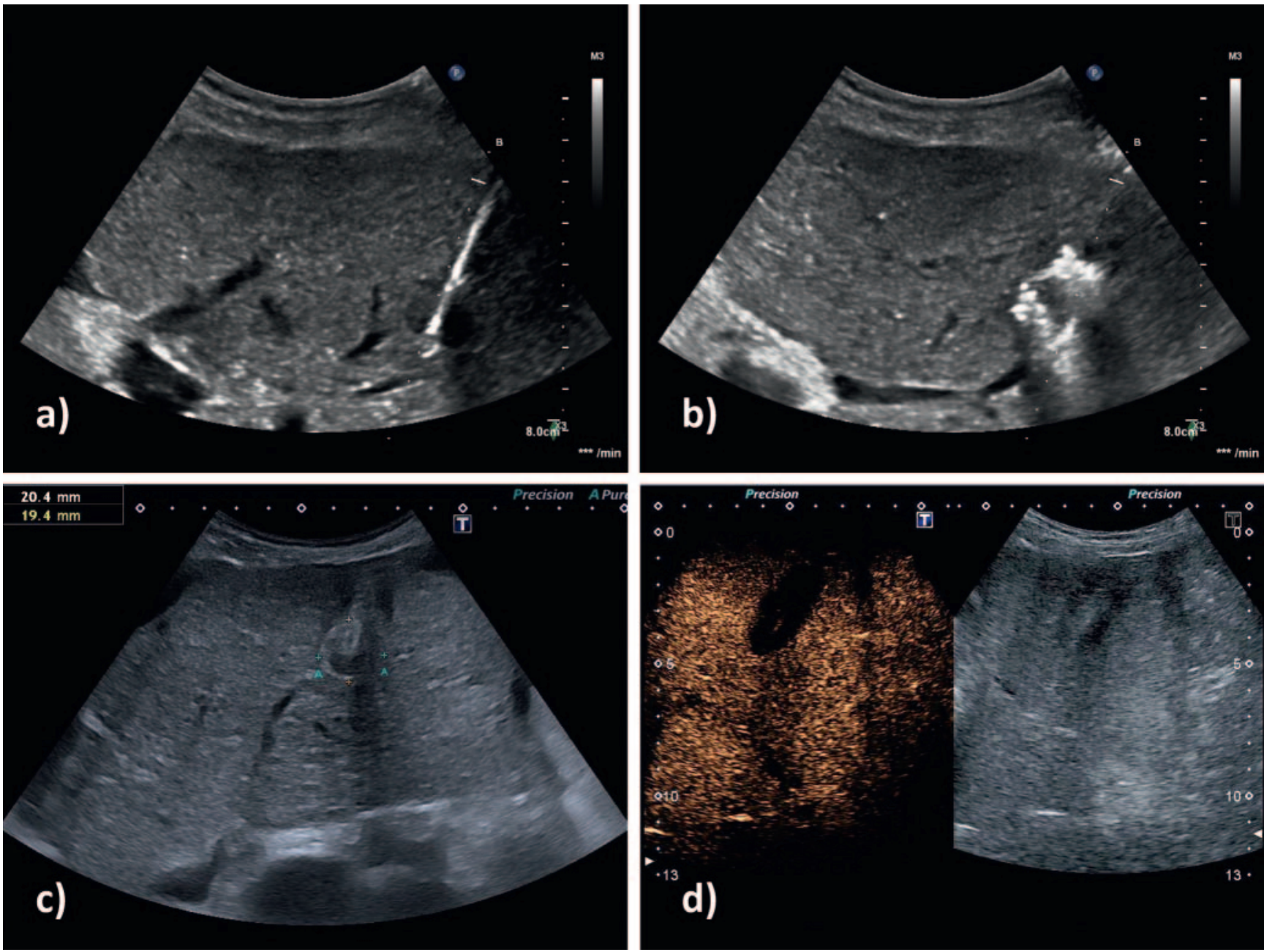

- Fig. 5 Microwave ablation of hepatocellular carcinoma (HCC). a positioning of the probe in the tumor $\mathbf{b}$ local gas formation during ablation c tumor after ablation in the B-scan $\mathbf{d}$ monitoring of the ablation area in contrast-enhanced ultrasound.

the generation of normal values and cut-off limits for liver stiffness as well as the comparability with already established methods is often still poor [39]. One current study including 4 different ultrasound devices was able to show high comparability of shear wave velocities but only in healthy subjects [40] ( $\triangleright$ Fig. 4). The determination of tissue elasticity in untreated chronic hepatitis $B$ and $C$ to rule out significant fibrosis and cirrhosis is currently considered a definite indication. Liver stiffness typically decreases under antiviral treatment with analogs. Screening for possible hepatocellular carcinoma and portal hypertension should be performed in patients with advanced disease regardless of decreasing liver stiffness. Liver stiffness cannot be used to rule out liver fibrosis or cirrhosis in viremic patients due to the lack of cutoff values. Moreover, elastography can be used in non-alcoholic fatty liver disease, alcoholic liver disease, and suspicion of liver cirrhosis to rule out advanced fibrosis [40]. The significance of liver stiffness in hepatic steatosis has not yet been definitively clarified. This must be taken into consideration when interpreting the liver stiffness results in patients with severe steatosis and obesity [41]. When performing elastography, nutritional status, respiration (examination should not be performed during inspiration), liver val- ues 5 times higher than normal, cholestasis, liver congestion, and infiltrative liver diseases can affect results. For several years spleen stiffness measurement particularly for evaluating prognosis in esophageal varices and as a marker for portal hypertension has increasingly become the focus of scientific studies [42, 43]. Due to the contradictory data, spleen elastography cannot yet be categorized as an established method [44]. Other extrahepatic applications like elastography of the pancreas are currently the subject of scientific studies [45].

\section{Microwave therapy}

\section{Technique}

Various local ablative methods are used in tumor therapy [46]:

- Radiofrequency ablation (RFA)

- Microwave ablation (MWA)

- Laser-induced interstitial thermotherapy (LITT)

- Irreversible electroporation (IRE)

- Cryoablation. 
Microwave ablation (MWA) was developed in the $1980 \mathrm{~s}$ from an intraoperative method for achieving hemostasis [46]. Numerous providers and various types of devices are currently available on the market. In addition to use in CT, MWA can also be performed under ultrasound guidance. Compared to other methods, MWA has advantages in large tumor areas and areas close to large vessels. Microwave energy quickly generates high temperatures with a greater kinetic energy than RFA [47]. MWA is less susceptible to the heat sink effect caused by adjacent vessels than the well established RFA method. Microwave ablation (MWA) is based on the emission of electromagnetic waves whose alternating field causes local water molecules to vibrate. A spherical ablation area is created around the tip of the probe. Heat is transmitted to the tissue in a centrifugal fashion around the tip of the probe. This causes denaturation of the surrounding (tumor) cells resulting in an ablation zone.

\section{Clinical applications}

The most common application site is the liver both for primary liver tumors like HCCs and for secondary liver tumors like metastases [46] ( $\triangleright$ Fig. 5). Successful application of MWA for pancreatic neoplasms and renal cell carcinomas (T1a or smaller T1b) has also been described $[48,49]$. A current meta-analysis examining radiofrequency ablation and microwave ablation compared to systemic chemotherapy and partial hepatectomy in the treatment of colorectal liver metastases was able to show an advantage of ablation methods compared to chemotherapy [50]. To our knowledge, there are currently no studies examining microwave ablation using ultrasound guidance vs. CT guidance.

\section{Conclusion}

With their broad spectrum of long established as well as newer modalities, ultrasound methods are a useful addition to CT and MRI. In particular, contrast-enhanced ultrasound can greatly expand the diagnostic spectrum in radiology. New microvascular Doppler ultrasound methods make it possible to eliminate the administration of contrast agent and the use of ionizing radiation in patients who can be effectively examined with ultrasound. Sonoelastography methods are additional tools in the diagnostic arsenal for diagnosing fibrotic liver diseases without the use of invasive methods. Fusion imaging is currently still a niche method. However, with increasing development of the technology, image fusion can be expected to become increasingly important in diagnostic imaging in the future.

Due to the complexity of the new ultrasound methods, they present a growing challenge in training and continuing education and cannot be sufficiently represented or learned using current continuing education concepts. The necessary competence can presumably be better acquired in interdisciplinary ultrasound units. However, these are currently not sufficiently available.

As a complement to the existing and well established DEGUM level concept, a targeted change to the Regulation on Continuing Education with the introduction of a corresponding additional qualification in diagnostic ultrasound imaging could increase the interest of younger colleagues in ultrasound within radiology. The acquired competences could also be used for subsequent further professional development.

\section{Financial support}

None.

\section{Conflict of Interest}

The authors declare that they have no conflict of interest.

\section{References}

[1] Graeter T, Kratzer W, Seufferlein T et al. Evaluation of the value of contrast-enhanced ultrasound (CEUS) within radiology departments in germany. Rofo 2017; 189: 748-759. doi:10.1055/s-0043-111011

[2] Gebündelte Kompetenz statt zergliederter Fuhrpark, Vortrag Prof. T. F. Fischer, Deutsche Röntgengesellschaft. 2016

[3] Ferraioli G, Meloni MF. Contrast-enhanced ultrasonography of the liver using SonoVue. Ultrasonography 2018; 37: 25-35. doi:10.14366/ usg. 17037

[4] Piscaglia F, Bolondi L. Italian Society for Ultrasound in Medicine and Biology (SIUMB) Study Group on Ultrasound Contrast Agents.. The safety of sonovue in abdominal applications: Retrospective analysis of 23188 investigations. Ultrasound Med Biol 2006; 32: 1369-1375. doi:10.1016/ j.ultrasmedbio.2006.05.031

[5] Strobel D, Seitz K, Blank W et al. Contrast-enhanced ultrasound for the characterization of focal liver lesions-diagnostic accuracy in clinical practice (DEGUM multicenter trial). Ultraschall in Med 2008; 29: 499505. doi:10.1055/s-2008-1027806

[6] Thaiss WM, Bedke J, Kruck S et al. Can contrast-enhanced ultrasound and acoustic radiation force impulse imaging characterize $\mathrm{CT}$-indeterminate renal masses? A prospective evaluation with histological confirmation. World J Urol 2019; 37: 1339-1346. doi:10.1007/s00345-018-2520-3

[7] Jäschke M, Weber MA, Fischer C. [CEUS-application possibilities in the musculoskeletal system]. Radiologe 2018; 58: 579-589. doi:10.1007| s00117-018-0404-6

[8] Fischer C, Haug T, Weber MA et al. Contrast-enhanced ultrasound (CEUS) identifies perfusion differences between tibial fracture unions and non-unions. Ultraschall in Med 2018. doi:10.1055/a-0720-1610

[9] Doll ], Gross S, Weber MA et al. The AMANDUS project-advanced microperfusion assessed non-union diagnostics with contrast-enhanced ultrasound (CEUS) for the detection of infected lower extremity non-unions. Ultrasound Med Biol 2019; 45: 2281-2288. doi:10.1016/j.ultrasmedbio.2019.05.007

[10] Marcon J, Trottmann M, Stief CG et al. CEUS-use in testicular pathologies. Radiologe 2018; 58: 57-578. doi:10.1007/s00117-018-0387-3

[11] Rübenthaler ], Reiser M, Cantisani $V$ et al. The value of contrast-enhanced ultrasound (CEUS) using a high-end ultrasound system in the characterization of endoleaks after endovascular aortic repair (EVAR). Clin Hemorheol Microcirc 2017; 66: 283-292. doi:10.3233/CH-179102

[12] Goeral K, Hojreh A, Kasprian G et al. Microvessel ultrasound of neonatal brain parenchyma: Feasibility, reproducibility, and normal imaging features by superb microvascular imaging (SMI). Eur Radiol 2019; 29: 2127-2136. doi:10.1007/s00330-018-5743-1

[13] Jiang ZZ, Huang YH, Shen HL et al. Clinical applications of superb microvascular imaging in the liver, breast, thyroid, skeletal muscle, and carotid plaques. J Ultrasound Med 2019; 38: 2811-2820. doi:10.1002/ jum. 15008 
[14] Dubinsky T], Revels J, Wang S et al. Comparison of superb microvascular imaging with color flow and power doppler imaging of small hepatocelIular carcinomas. J Ultrasound Med 2018; 37: 2915-2924. doi:10.1002/ jum.14654

[15] Sim JK, Lee JY, Hong HS. Differentiation between malignant and benign lymph nodes: Role of superb microvascular imaging in the evaluation of cervical lymph nodes. J Ultrasound Med 2019; 38: 3025-3036. doi:10.1002/jum. 15010

[16] Mao Y, Mu J, Zhao J et al. The value of superb microvascular imaging in differentiating benign renal mass from malignant renal tumor: A retrospective study. Br J Radiol 2018; 91: 20170601. doi:10.1259/ bjr.20170601

[17] Mu J, Mao Y, Li F et al. Superb microvascular imaging is a rational choice for accurate bosniak classification of renal cystic masses. Br J Radiol 2019; 92: 20181038. doi:10.1259/bjr.20181038

[18] Lu R, Meng Y, Zhang Y et al. Superb microvascular imaging (SMI) compared with conventional ultrasound for evaluating thyroid nodules. BMC Med Imaging 2017; 17: 65. doi:10.1186/s12880-017-0241-5

[19] Gabriel M, Tomczak J, Snoch-Ziółkiewicz M et al. Superb micro-vascular imaging (SMI): A doppler ultrasound technique with potential to identify, classify, and follow up endoleaks in patients after endovascular aneurysm repair (EVAR). Abdom Radiol (NY) 2018; 43: 3479-3486. doi:10.1007/s00261-018-1633-x

[20] Ra JC, Lee ES, Park H] et al. Efficacy of superb microvascular imaging for diagnosing acute cholecystitis: Comparison with conventional ultrasonography. Ultrasound Med Biol 2018; 44: 1968-1977. doi:10.1016/ j.ultrasmedbio.2018.05.014

[21] Park AY, Seo BK, Woo OH et al. The utility of ultrasound superb microvascular imaging for evaluation of breast tumour vascularity: Comparison with colour and power doppler imaging regarding diagnostic performance. Clin Radiol 2018; 73: 304-311. doi:10.1016/ j.crad.2017.10.006

[22] Chen J, Chen L, Wu L et al. Value of superb microvascular imaging ultrasonography in the diagnosis of carpal tunnel syndrome: Compared with color doppler and power doppler. Medicine (Baltimore) 2017; 96: e6862. doi:10.1097/MD.0000000000006862

[23] Durmaz MS, Sivri M. Comparison of superb micro-vascular imaging (SMI) and conventional doppler imaging techniques for evaluating testicular blood flow. J Med Ultrason (2001) 2018; 45: 443-452. doi:10.1007/s10396-017-0847-9

[24] Karaca L, Oral A, Kantarci M et al. Comparison of the superb microvascular imaging technique and the color doppler techniques for evaluating children's testicular blood flow. Eur Rev Med Pharmacol Sci 2016; 20: 1947-1953

[25] Jung EM, Clevert DA. [Possibilities of sonographic image fusion: Current developments]. Radiologe 2015; 55: 937-948. doi:10.1007| s00117-015-0025-2

[26] Ewertsen C, Henriksen BM, Torp-Pedersen S et al. Characterization by biopsy or CEUS of liver lesions guided by image fusion between ultrasonography and CT, PET/CT or MRI. Ultraschall in Med 2011; 32: 191-197. doi:10.1055/s-0029-1245921

[27] Okamoto E, Sato S, Sanchez-Siles AA et al. Evaluation of virtual CT sonography for enhanced detection of small hepatic nodules: A prospective pilot study. Am J Roentgenol 2010; 194: 1272-1278. doi:10.2214/Am J Roentgenol.08.2294

[28] Rübenthaler J, Paprottka KJ, Marcon J et al. MRI and contrast enhanced ultrasound (CEUS) image fusion of renal lesions. Clin Hemorheol Microcirc 2016; 64: 457-466. doi:10.3233/CH-168116

[29] Jung EM, Clevert DA. [Contrast-enhanced ultrasound (CEUS) and image fusion for procedures of liver interventions]. Radiologe 2018; 58: 538544. doi:10.1007/s00117-018-0411-7
[30] Lee MW. Fusion imaging of real-time ultrasonography with CT or MRI for hepatic intervention. Ultrasonography 2014; 33: 227-239. doi:10.14366/usg. 14021

[31] Jung EM, Uller W, Stroszczynski C et al. Contrast-enhanced sonography. therapy control of radiofrequency ablation and transarterial chemoembolization of hepatocellular carcinoma. Radiologe 2011; 51: 462-468. doi:10.1007/s00117-010-2101-y

[32] Kang TW, Lee MW, Cha DI et al. Usefulness of virtual expiratory CT images to compensate for respiratory liver motion in Ultrasound/CT image fusion: A prospective study in patients with focal hepatic lesions. Korean J Radiol 2019; 20: 225-235. doi:10.3348/kjr.2018.0320

[33] Maxeiner A, Fischer T, Schwabe J et al. Contrast-Enhanced Ultrasound (CEUS) and Quantitative Perfusion Analysis in Patients with Suspicion for Prostate Cancer. Ultraschall in Med 2019; 40: 340-348. doi:10.1055/ a-0594-2093

[34] Chang S, Kim M-J, Kim J et al. Variability of Shear Wave Velocity using Different Frequencies in Acoustic Radiation Force Impulse (ARFI) Elastography: A Phantom and Normal Liver Study. Ultraschall der Medizin - Eur J Ultrasound 2012: 260-265. doi:10.1055/s-0032-1313008

[35] Sporea I, Bota S, Jurchis A et al. Acoustic radiation force impulse and supersonic shear imaging versus transient elastography for liver fibrosis assessment. Ultrasound Med Biol 2013; 39: 1933-1941. doi:10.1016/ j.ultrasmedbio.2013.05.003

[36] Bamber J, Cosgrove D, Dietrich CF et al. EFSUMB guidelines and recommendations on the clinical use of ultrasound elastography. Part 1: Basic Principles and Technology. Ultraschall in Med 2013; 34: 169-184. doi:10.1055/s-0033-1335205

[37] Friedrich-Rust M, Nierhoff J, Lupsor M et al. Performance of Acoustic Radiation Force Impulse imaging for the staging of liver fibrosis: A pooled meta-analysis. J Viral Hepat 2012; 19: 212-219. doi:10.1111/ j.1365-2893.2011.01537.x

[38] Cassinotto C, Lapuyade B, Mouries A et al. Non-invasive assessment of liver fibrosis with impulse elastography: Comparison of Supersonic Shear Imaging with ARFI and FibroScan ${ }^{\circledR}$. J Hepatol 2014; 61: 550-557. doi:10.1016/j.jhep.2014.04.044

[39] Piscaglia F, Salvatore V, Mulazzani L et al. Ultrasound Shear Wave Elastography for Liver Disease. A Critical Appraisal of the Many Actors on the Stage. Ultraschall in Med 2016; 37: 1-5. doi:10.1055/s-0035-1567037

[40] Ferraioli G, Wong VW, Castera L et al. Liver Ultrasound Elastography: An Update to the World Federation for Ultrasound in Medicine and Biology Guidelines and Recommendations. Ultrasound Med Biol 2018; 44: 2419-2440. doi:10.1016/j.ultrasmedbio.2018.07.008

[41] Dietrich CF, Bamber J, Berzigotti A et al. EFSUMB Guidelines and Recommendations on the Clinical Use of Liver Ultrasound Elastography, Update 2017 (Long Version). Ultraschall in Med 2017; 38: e48. doi:10.1055/a-0641-0076

[42] Gibiino G, Garcovich M, Ainora ME et al. Spleen ultrasound elastography: state of the art and future directions - a systematic review. Eur Rev Med Pharmacol Sci 2019; 23: 4368-4381. doi:10.26355/eurrev_201905_17944

[43] Săftoiu A, Gilja OH, Sidhu PS et al. The EFSUMB Guidelines and Recommendations for the Clinical Practice of Elastography in Non-Hepatic Applications: Update 2018. Ultraschall in Med 2019; 40: 425-453. doi:10.1055/a-0838-9937

[44] Cho YS, Lim S, Kim Y et al. Spleen Stiffness Measurement Using 2-Dimensional Shear Wave Elastography: The Predictors of Measurability and the Normal Spleen Stiffness Value. J Ultrasound Med 2019; 38: 423-431. doi:10.1002/jum. 14708

[45] Kloth C, Fabricius D, Wendlik I et al. Diagnostic accuracy of MRI with MRCP and B-Mode-sonography with elastography of the pancreas in patients with cystic fibrosis: a point-to-point comparison. BMC Res Notes 2019; 12: 150. doi:10.1186/s13104-019-4193-4 
[46] Vogl T], Nour-Eldin NA, Hammerstingl RM et al. Microwave ablation (MWA): Basics, technique and results in primary and metastatic liver neoplasms - review article. Rofo 2017; 189: 1055-1066. doi:10.1055/ s-0043-117410

[47] Facciorusso A, Di Maso M, Muscatiello N. Microwave ablation versus radiofrequency ablation for the treatment of hepatocellular carcinoma: A systematic review and meta-analysis. Int J Hyperthermia 2016; 32: 339-344. doi:10.3109/02656736.2015.1127434

[48] Vogl T], Panahi B, Albrecht MH et al. Microwave ablation of pancreatic tumors. Minim Invasive Ther Allied Technol 2018; 27: 33-40. doi:10.1080/13645706.2017.1420664
[49] Welch BT, Shah PH, Thompson RH et al. The current status of thermal ablation in the management of T1b renal masses. Int J Hyperthermia 2019; 36: 31-36. doi:10.1080/02656736.2019.1605097

[50] Meijerink MR, Puijk RS, van Tilborg AAJM et al. Radiofrequency and Microwave Ablation Compared to Systemic Chemotherapy and to Partial Hepatectomy in the Treatment of Colorectal Liver Metastases: A Systematic Review and Meta-Analysis. Cardiovasc Intervent Radiol 2018; 41: 1189-1204. doi:10.1007/s00270-018-1959-3 